\title{
AVALIAÇÃO DAS PROPRIEDADES PSICOMÉTRICAS BÁSICAS PARA A VERSÃO EM PORTUGUÊS DO KDQOL-SF ${ }^{T M^{*}}$
}

\author{
Cleide Aparecida Moreira', Walter Garletti Junior', Lucimara Ferreira lima', Cristiane Rodrigues lima', Juliana Facunde Ribeiro',
} Alba Franzon Miranda*2

Trabalho realizado na Clínica Neonefro Nefrologia e Clínica Médica, Indaiatuba, SP

*Correspondência:

Rua Dr. Quirino, 1577

apto 71

CEP 13015-082

Campinas - SP

albamira@icm.unicamp.br

\section{RESUMO}

OBJEtivo. Verificar a confiabilidade do instrumento Kidney Disease Quality of Life Short Form - KDQOL-SF e correlacionar os escores em cada uma das dimensões com variáveis sociodemográficas e clínicas.

MéTodos. Trata-se de um estudo metodológico exploratório descritivo, no qual o KDQOL-SF foi aplicado por meio de entrevista em 147 pacientes renais crônicos em tratamento em uma clínica de hemodiálise, no interior do Estado de São Paulo. A análise dos dados foi por meio de estatística descritiva, $\alpha$ de Cronbach e análise de regressão logística ordinal.

Resultados. Os resultados mostraram que dos 147 sujeitos que responderam ao KDQOL-SF, a maioria era do sexo masculino, com idade média de 51,8 \pm 14,2 anos e 75,5\% haviam cursado apenas o ensino fundamental. Em relação à confiabilidade do KDQOL-SF, verificada por meio do $\alpha$ de Cronbach, os valores variaram entre 0,6 e 1,0 para 18 das 19 dimensões que compõem o instrumento. Apenas a dimensão 'papel profissional' mostrou um valor extremamente baixo. Quando o KDQOL-SF foi correlacionado com as variáveis sociodemográficas e clínicas, houve associação de algumas dimensões com a idade, sexo, grau de escolaridade e patologias associadas à doença renal.

ConcLusão. O KDQOL-SF mostrou-se confiável para verificar a qualidade de vida em indivíduos com IRC em tratamento hemodialítico.

UNITERMOS: Qualidade de vida. Insuficiência renal crônica. Diálise renal.

\section{INTRODUÇÃO}

O conceito qualidade de vida $(\mathrm{QV})$, principalmente voltado à terapêutica assistencial cresce e sua operacionalização ocorre por meio da criação de instrumentos que avaliam e mensuram essa variável em seus vários aspectos ${ }^{1,2,3,4}$. A QV é uma variável subjetiva e vem sendo mensurada de modo que se possa ter uma leitura objetiva do seu significado para os sujeitos, dentro da sua própria visão de QV 5,6 Os aspectos subjetivos mensurados são percepções envolvidas que abrangem as condições físicas, psicológicas, sociais, culturais e espirituais sob o ponto de vista dos indivíduos?.

O termo QV assume importância para portadores de doenças crônicas, por estas patologias possuírem aspecto negativo na vida dessas pessoas. Dessa forma, os instrumentos de avaliação e mensuração da QV tornam-se cada vez mais completos e específicos ${ }^{7.8}$. Estes instrumentos apresentam divididos em genéricos e específicos, sendo que os primeiros são os que avaliam a QV de maneira ampla em uma população, enquanto os específicos são instrumentos destinados a mensurar QV em sujeitos que possuem as mesmas características de uma determinada patologia ${ }^{8,9,10,11}$.
Esses instrumentos de avaliação de QV são, em sua maioria, concretizados em países estrangeiros e para utilizá-los em outros países que não os de sua origem devem passar por um processo de tradução e adaptação transcultural ao idioma do país no qual se pretende aplicá-

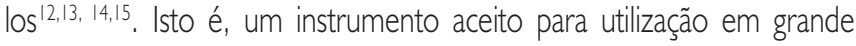
escala, após sua tradução e adaptaçãa, deve passar por um processo de avaliação de suas propriedades psicométricas. Portanto, as ferramentas que foram desenvolvidas por outros pesquisadores devem ser testadas de maneira a identificar se são medidas válidas e confiáveis. A confiabilidade e a validade, quando demonstradas, influenciam profundamente a robustez das descobertas de um estudo ${ }^{16,17,18}$

Dessa maneira, a avaliação da qualidade de vida, por meio desses instrumentos, pode fornecer um perfil das necessidades de cuidados e intervenções para esses indivíduos, sobre os quais seria possível organizar os conhecimentos necessários na área, além de permitir comparações entre populações e intervenções específicas à clientela.

Visando avaliar as propriedades psicométricas básicas do instrumento específico, Kidney Disease Quality of Life Short Form - KDQOL-SF, o presente estudo teve como objetivos: verificar a confiabilidade do 
KDQOL-SF e correlacionar os escores obtidos em cada uma das suas 19 dimensões com as variáveis sociodemográficas e clínicas.

\section{Métodos}

Trata-se de um estudo metodológico exploratório descritivo realizado em uma clínica de hemodiálise, na cidade de Indaiatuba, no interior do Estado de São Paulo.

O projeto foi iniciado após a aprovação pelo Comitê de Ética em Pesquisas da Universidade Paulista, sob o número 21/06. Todos os sujeitos assinaram o termo de consentimento livre e esclarecido.

De um total de 160 sujeitos em tratamento hemodiálitico, a amostra ficou composta por 147 pacientes de ambos os sexos, portadores de IRC. Treze indivíduos foram excluídos do estudo por: quatro por óbito; três por pertencerem a um outro programa de diálise; quatro por apresentarem transtornos psicológicos; e dois por não concordarem em participar pesquisa.

Utilizou-se na coleta dos dados dois instrumentos: um elaborado especificamente para coletar as variáveis sociodemográficas (idade, sexo, grau de escolaridade, condições de habitação (foram avaliados, de maneira subjetiva, a localização, tipo de construção, número de cômodos/habitantes e saneamento básico), ocupação atual, atividade de lazer) e clínicas (tempo de hemodiálise, patologias associadas à IRC, valores de hemoglobina, albumina, ureia pré e pós-hemodiálise, creatinina); e outro para avaliar a qualidade de vida em portadores de IRC, o KDQOL-SF. Esse instrumento é composto por 80 itens, que incluem o Short Form - 36 Item Health Survey (SF-36) e mais 43 itens sobre a doença renal crônica. OSF-36 é composto de 36 itens divididos em oito dimensões: funcionamento físico ( 10 itens), função física (quatro itens), função emocional (três itens), função social (dois itens), bem-estar emocional (cinco itens), dor (dois itens), energia /fadiga (quatro itens), saúde geral (cinco itens). O estado da saúde atual comparando há um ano (um item) e avaliação da saúde no geral (um item) são computados à parte. $O$ módulo espećfico sobre a doença renal inclui itens divididos em I I dimensões: sintomas / problemas ( 12 itens), efeito da doença renal (oito itens), sobrecarga da doença renal (quatro itens), papel profissional (dois itens), função cognitiva (três itens), qualidade da interação social (três itens), função sexual (dois itens) e sono (quatro itens). Estão incluídas no KDQOL-SF três escalas adicionais: suporte social (dois itens), estímulo por parte da equipe de diálise (dois itens) e satisfação do paciente (um item).

Para obter uma pontuação final do KDQOL-SF, os dados brutos devem ser recodificados em uma escala de $0-100$, de modo que os escores mais altos sempre refletem melhor qualidade de vida. Os itens são distribuídos entre as I| dimensões, seguindo orientações dos autores da versão para o português do KDQOL-SF. Após, as pontuações de cada dimensão são somadas para produzirem o escore de cada uma. O escore final da dimensão será a média para todos os itens respondidos pertencentes à mesma.

Os dados da pesquisa foram obtidos por meio de entrevistas e consultas nos prontuários dos pacientes. Tais procedimentos foram realizados pelos próprios pesquisadores. O tempo médio de duração das entrevistas para a aplicação do KDQOL-SF foi de $23 \pm 10$ minutos.
Os dados, após coletados, foram categorizados quando se tratavam de variáveis nominais, transportados para planilha de dados do programa Excel for Windows/2003 e depois para o software Statistical Package for Social Science (SPSS-13). As seguintes análises foram realizadas com assessoria de empresa de estatística vinculada à Universidade Estadual de Campinas - UNICAMP: de consistência interna com emprego do coeficiente $\alpha$ de Cronbach; e de regressão logística ordinal, para verificar a associação entre as variáveis sociodemográficas e clínicas; e os escores das 19 dimensões do KDQOL-SF. Fixou-se em 0,05 o nível de significância para as correlações.

Os valores do coeficiente $\alpha$ de Cronbach variam de zero a um, sendo que quanto mais próximo de um, maior será a consistência interna. Como regra geral, recomenda-se valor de alfa acima de 0,80 para uma escala amplamente utilizada, embora valores acima de 0,60 já indiquem consistência' ${ }^{19}$.

\section{Resultados}

Conforme apresentado na Tabela I, a maioria dos participantes era do sexo masculino, com idade média de 51,8 anos e em tratamento hemodialítico há aproximadamente dois anos e meio. Os participantes caracterizaram-se predominantemente pela baixa escolaridade, mais de $50 \%$ eram inativos, devido a $30,6 \%$ já terem se aposentado, e 27,2\% não terem atividades laborais. Mais de 70\% dos entrevistados declararam praticar atividades de lazer, descritas como caminhadas, assistir programas de TV, leitura, visitar familiares e/ou amigos e viagens apenas por curto período entre as sessões de hemodiálise. Sobre as condições de moradia, considerou-se boa para a maioria das pessoas. A patologia associada prevalente nesta população foi hipertensão arterial sistêmica (HAS) presente em 43,2\% dos sujeitos, seguida de Diabetes mellitus (DM) em 4I, 1\%. Os valores médios dos exames laboratoriais foram compatíveis às pessoas com insuficiência renal crônica.

Os valores do $\alpha$ de Cronbach para cada uma das dimensões do KDQOL-SF são apresentados na Tabela 2. Esses valores variaram entre 0,6 e I,0 com exceção da dimensão 'papel profissional', que mostrou valores aproximados de 0,1 .

Os escores médios das dimensões do KDQOL-SF também são apresentados na Tabela 2, sendo que os menores valores obtidos foram nas dimensões 'sobrecarga da doença renal', 'papel profissional' e 'função sexual' para o módulo espećfico. Os escores médios mais baixos, para o módulo que avalia a QV de forma genérica (SF-36), foram nas dimensões 'função física' e 'função emocional'.

A Tabela 3 explora a relação entre o que foi respondido pelo participante e as variáveis sociodemográficas e clínicas. Quando as 19 dimensões do KDQOL-SF foram correlacionadas com essas variáveis, observou-se correlação entre o sexo masculino e a dimensão 'dor', isto é, as pessoas do sexo masculino apresentaram maiores escores nesta dimensão quando comparadas as do sexo feminino. A idade correlacionou-se com as dimensões 'sono', 'funcionamento físico' e 'dor', de maneira que quando a idade foi acrescida em uma unidade, as chances aumentaram para os sujeitos apresentarem pontuação mais baixa nestas dimensões. $\mathrm{O}$ grau de escolaridade, subdividido em $1^{\circ}, 2^{\circ}$, $3^{\circ}$ grau completo /incompleto e pós-graduação, mostrou correlação, 
Moreira CA et al.

Tabela I - Características sociodemográficas e clínicas dos 147 pacientes em tratamento hemodiálitico. Indaiatuba - SP, 2006

\begin{tabular}{|c|c|c|}
\hline Características & $\mathrm{n}(\%)$ & Média $\pm \mathrm{DP}$ \\
\hline $\begin{array}{l}\text { Idade (anos) } \\
\text { Tempo HD (meses) }\end{array}$ & & $\begin{array}{l}51,8 \pm 14,2 \\
28,6 \pm 30,5\end{array}$ \\
\hline $\begin{array}{l}\text { Sexo } \\
\text { Feminino } \\
\text { Masculino }\end{array}$ & $\begin{array}{l}71(48,3) \\
76(51,4)\end{array}$ & \\
\hline $\begin{array}{l}\text { Grau de escolaridade } \\
1^{\circ} \text { grau completo/incompleto } \\
2^{0} \text { grau completo/incompleto } \\
3^{0} \text { grau completo/incompleto } \\
\text { Pós-graduação }\end{array}$ & $\begin{array}{l}111(75,5) \\
26(17,7) \\
9(6,1) \\
1(0,7)\end{array}$ & \\
\hline $\begin{array}{l}\text { Ocupação atual } \\
\text { Trabalha com remuneração } \\
\text { Não trabalha } \\
\text { Aposentado } \\
\text { Do lar }\end{array}$ & $\begin{array}{l}23(15,7) \\
40(27,2) \\
45(30,6) \\
39(26,5)\end{array}$ & \\
\hline $\begin{array}{l}\text { Atividades de lazer } \\
\text { Tem alguma atividade de lazer } \\
\text { Não tem atividade de lazer }\end{array}$ & $\begin{array}{l}110(74,8) \\
37(25,2)\end{array}$ & \\
\hline $\begin{array}{l}\text { Condições de habitação } \\
\text { Ruim } \\
\text { Boa } \\
\text { Muito Boa }\end{array}$ & $\begin{array}{c}8(5,4) \\
\mid 35(9 \mid, 8) \\
4(2,8)\end{array}$ & \\
\hline $\begin{array}{l}\text { Patologias associadas } \\
\text { DM } \\
\text { HAS } \\
\text { Lúpus heritematoso } \\
\text { DM+HAS } \\
\text { Outras }\end{array}$ & $\begin{array}{l}15(10,6) \\
6 \mid(43,2) \\
I(0,7) \\
58(41,1) \\
6(4,4)\end{array}$ & \\
\hline $\begin{array}{l}\text { Albumina }(\mathrm{g} / \mathrm{dl}) \\
\text { Hemoglobina }(\mathrm{g} / \mathrm{dl}) \\
\text { Ureia pré-HD (mg/dl) } \\
\text { Ureia pós HD (mg/dl) } \\
\text { Creatinina }(\mathrm{mg} / \mathrm{dl})\end{array}$ & & $\begin{array}{c}4,2 \pm 0,5 \\
10,4 \pm 2,9 \\
145,1 \pm 34,9 \\
49,6 \pm 18,7 \\
9,8 \pm 3,2\end{array}$ \\
\hline
\end{tabular}

$\mathrm{DM}=$ diabetes mellitus $; \mathrm{HSA}=$ hipertensão arterial sistêmica; $\mathrm{HD}=$ hemodiálise

apenas, entre a categoria $2^{\circ}$ grau completo/incompleto e 'sobrecarga da doença renal', isto é, os sujeitos com $2^{\circ}$ grau completo/incompleto mostraram chances maiores de responder com escores mais elevados, nessa dimensão, quando comparados aos sujeitos com $1^{\circ}$ grau de escolaridade.

A boa condição de habitação apresentou correlação com as dimensões 'suporte social' e 'dor', sendo que 'energia/ fadiga' mostrou associação com condições de habitação muito boa, ambas comparadas à categoria 'condições ruins de moradia'. Assim, as pessoas com melhores condições de habitação apresentaram chances maiores de responder com escores mais elevados nas dimensões 'suporte social', 'dor' e 'energia/fadiga'.

Quando os sujeitos foram avaliados em relação à ocupação atual, pôde-se notar que houve correlação entre as respostas dadas pelas pessoas que não trabalham e as dimensões 'suporte social', 'estímulo por parte da equipe' e 'papel profissional'. Dessa maneira, as pessoas que não trabalham apresentaram chances maiores de pontuar com escores mais elevados nas dimensões 'suporte social' e 'estímulo por parte da equipe', e escores mais baixos na dimensão 'papel profissional', quando comparadas àquelas que trabalham com remuneração.

Em relação à variável 'atividade de lazer', houve correlação entre a categoria não ter atividade de lazer e as dimensões 'funcionamento físico' e 'energia/fadiga'; de modo que as chances dos sujeitos que não possuem atividades de lazer foram maiores para responder com escores mais baixos nas dimensões 'funcionamento físico' e 'energia ffadiga' quando comparados aos sujeitos que possuem alguma atividade de lazer.

Quanto às patologias associadas, houve correlação entre a HAS com as dimensões 'sintomas/ problemas' e 'sobrecarga da doença renal'. Isto é, os sujeitos com HAS apresentaram chances maiores de responderem com pontuação mais baixa na dimensão 'sintomas e problemas' e com escores mais elevados na 'sobrecarga da doença renal' quando comparados com os sujeitos que são portadores apenas de DM. No entanto, quando as duas patologias estão associadas, a correlação foi com a dimensão 'bem-estar emocional', de maneira que os sujeitos portadores de HAS e DM têm chances elevadas de responder com escores mais baixos na dimensão 'bem-estar emocional' quando comparados às pessoas portadoras apenas de DM. O tempo em hemodiálise correlacionou-se com a dimensão 'função cognitiva', sendo que, ao aumentar o tempo de hemodiálise, os sujeitos apresentaram chances maiores de pontuar com escore mais alto na 'função cognitiva'. Em relação aos valores de albumina, estes mostraram correlação com a dimensão 'funcionamento físico', sendo que ao acrescer em uma unidade a esse valor, as chances das pessoas responderem com escores mais elevados aumentaram.

\section{DIscussão}

Os resultados do presente estudo mostraram que os índices de consistência interna do KDQOL- SF, verificado por meio do $\alpha$ de Cronbach, são aceitáveis, pois apontam valores que variam de 0,6 a I,0 com exceção na dimensão 'papel profissional'.

Para que uma ferramenta seja confável, o nível aceitável deve ser superior a $0,7^{18}$, porém valores acima de 0,6 são considerados para utilização de instrumentos em populações para os quais foram construídos. Valores abaixo de 0,6 indicam pouca consistência interna entre os itens ${ }^{18,19}$. Os valores extremamente baixos encontrados na dimensão 'papel profissional' mostraram, possivelmente, uma questão no entendimento dos sujeitos acarretando um problema na mensuração. Esse problema pode ser atribuído às respostas incongruentes dadas pelos sujeitos quando responderam a questão número 20 - durante as últimas quatro semanas não receberam dinheiro para trabalhar. No entanto, ao responderem a questão número 21 , consideraram que a sua saúde não os impossibilitava de ter um trabalho pago. Assim, para haver uma consistência entre as respostas das questões 20 e 21 , os sujeitos ao responderem não à primeira deveriam responder sim à segunda, respectivamente, porém também responderam não à questão 21 .

O entendimento dos sujeitos do estudo nessas duas questões que medem a dimensão 'papel profissional' não foi consistente, motivo pelo 
Tabela 2 - Valores médios dos escores dos I47 sujeitos com IRC em tratamento hemodialítico, e valores do $\alpha$ de Cronbach para cada uma das dimensões do KDQOL-SF. Indaiatuba - SP, 2006

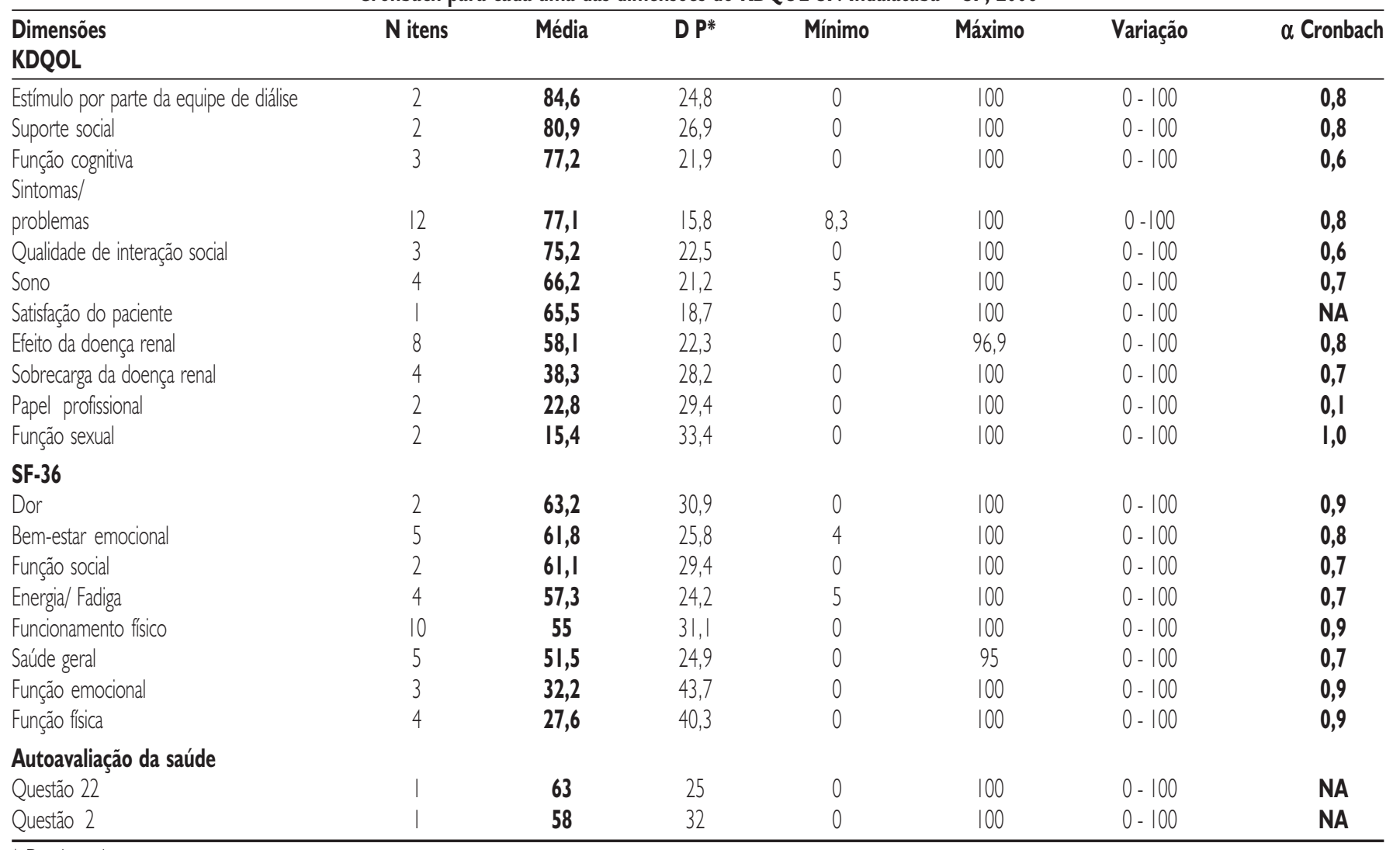

* Desvio padrão

- Questão 2 = Comparada há um ano atrás, como você avaliaria sua saúde em geral agora?

- Questão 22 = No geral, como você avaliaria sua saúde?

qual os valores de alfa foram extremamente baixos. Assim, a variação de erro nessa dimensão se mostrou muito alta, o que nos permite afirmar que a ferramenta tem um problema com erro de medição na dimensão 'papel profissional'18. No entanto, o valor baixo em uma de suas dimensões não compromete o instrumento como um todo.

Quando comparamos os valores do alfa aos encontrados por Penneger et al. ${ }^{20}$, nota-se que os valores foram, se não iguais, muito próximos, com exceção para dimensão 'papel profissional' e 'qualidade da interação social'. Acredita-se que tais achados possam ser devido às diferenças culturais. Segundo Minayo et al.', a noção de QV, que em último caso reflete o indivíduo, baseia-se em três pontos de referência: o histórico, o cultural e um aspecto que refere às estratificações ou classes sociais. Assim, a influência histórica na QV passa pelo desenvolvimento econômico, social e tecnológico, uma sociedade tem parâmetros diferentes de QV ao logo de sua história. A QV vista pelo aspecto cultural, leva em consideração os valores e necessidades construídos e hierarquizados diferentemente pelos povos, revelando suas tradições. E, no pilar das estratificações ou classes sociais, a QV vincula-se a diferenças também estratificadas, em que o bem-estar das camadas superiores é o que impera. A ascensão de uma camada inferior para uma superior traduz o conceito de QV!
O presente estudo foi comparado ao de Penneger et al. ${ }^{20}$, conduzido na Suíça, um país desenvolvido, que apresenta diferenças sociais, culturais e econômicas em relação ao Brasil. As diferenças encontradas nas dimensões 'papel profissional' e 'qualidade de interação social', nos dois estudos, vêm reforçar o poder discriminatório do KDQOL-SF em avaliar dimensões da QV entre povos. Porém, vários estudos têm avaliado as propriedades psicométricas do KDQOL-SF em vários países e os mesmos têm apontado que esse instrumento manifestou ser confível e válido em avaliar a QV dos indivíduos renais crônicos ${ }^{12,21,22,23,24} \mathrm{e}$ em pacientes que foram submetidos a um transplante renal ${ }^{25}$.

A média dos escores para cada uma das dimensões do instrumento KDQOL-SF mostrada na Tabela 2 demonstra que os menores valores obtidos foram nas dimensões 'sobrecarga da doença renal', 'papel profissional' e funções sexual, física e emocional. Os achados de escore médio do estudo de Pernneger et al. ${ }^{20}$ aproximaram-se aos resultados do presente estudo. Resultados semelhantes também foram encontrados por Kussomota ${ }^{26}$, quando avaliou a QV relacionada à saúde de pacientes em quatro unidades de hemodiálise, em uma cidade do interior do Estado de São Paulo. Isto nos permite afirmar que os pacientes com IRC, em tratamento hemodialítico, apresentam as dimensões 'papel profissional', 'sobrecarga da doença' e 'função física' 
Tabela 3 - Dimensões do KDQOL-SF que apresentaram correlaçōes significativas com as variáveis sociodemográficas e clínicas. Indaiatuba - SP, 2006

\begin{tabular}{|c|c|c|c|c|c|}
\hline $\begin{array}{l}\text { Variáveis sociodemográficas } \\
\text { e clínicas }\end{array}$ & Dimensões KDQOL-SF & $\begin{array}{l}\text { Coeficiente } \\
\text { correlação }\end{array}$ & $\mathbf{P}$ & OR & $\mathrm{IC} 95 \%$ \\
\hline Idade & $\begin{array}{c}\text { Sono } \\
\text { Funcionamento Físico } \\
\text { Dor }\end{array}$ & $\begin{array}{l}0,039 \\
0,045 \\
0,031\end{array}$ & $\begin{array}{l}0,000 \\
0,000 \\
0,007\end{array}$ & $\begin{array}{l}1,04 \\
1,50 \\
1,03\end{array}$ & $\begin{array}{l}1,02-1,06 \\
1,02-1,07 \\
1,01-1,06\end{array}$ \\
\hline $\begin{array}{l}\text { Sexo } \\
\text { Masculino } \\
\text { Feminino }\end{array}$ & Dor & $\begin{array}{l}1,119 \\
-\end{array}$ & 0,001 & 0,30 & $0,15-0,60$ \\
\hline $\begin{array}{l}\text { Grau de escolaridade } \\
1^{\circ} \text { Grau completo/incompleto } \\
2^{\circ} \text { Grau completo/incompleto } \\
3^{\circ} \text { Grau completo/incompleto } \\
\text { Pós-graduação }\end{array}$ & Sobrecarga da doença renal & $\begin{array}{l}- \\
-0,967 \\
- \\
-\end{array}$ & $\begin{array}{c}- \\
0,034 \\
- \\
-\end{array}$ & $\begin{array}{c}- \\
0,38 \\
-\end{array}$ & $\begin{array}{c}- \\
0,16-0,93 \\
-\end{array}$ \\
\hline $\begin{array}{l}\text { Condiçōes de habitação } \\
\text { Ruim } \\
\text { Boa } \\
\text { Muito boa }\end{array}$ & $\begin{array}{c}\text { Suporte Social } \\
\text { Dor } \\
\text { Energia /Fadiga }\end{array}$ & $\begin{array}{l}- \\
-1,560 \\
-1,568 \\
-2,547\end{array}$ & $\begin{array}{l}- \\
0,024 \\
0,029 \\
0,037-\end{array}$ & $\begin{array}{l}- \\
0,21 \\
0,21 \\
0,08-\end{array}$ & $\begin{array}{c}- \\
0,05-0,81 \\
0,05-0,85 \\
0,01-0,86-\end{array}$ \\
\hline $\begin{array}{l}\text { Ocupação atual } \\
\text { Trabalha com remuneração } \\
\text { Não trabalha }\end{array}$ & $\begin{array}{c}\text { Suporte Social } \\
\text { Estímulo por Parte da Equipe } \\
\text { Papel Profissional }\end{array}$ & $\begin{array}{l}- \\
-1,300 \\
-1,216 \\
1,839\end{array}$ & $\begin{array}{c}- \\
0,032 \\
0,027 \\
0,003\end{array}$ & $\begin{array}{l}- \\
0,27 \\
0,30 \\
6,29\end{array}$ & $\begin{array}{l}- \\
0,08-0,89 \\
0,10-0,87 \\
1,90-20,80\end{array}$ \\
\hline $\begin{array}{l}\text { Aposentado } \\
\text { Do lar }\end{array}$ & - & $\begin{array}{l}- \\
-\end{array}$ & $\begin{array}{l}- \\
-\end{array}$ & - & - \\
\hline $\begin{array}{l}\text { Atividade de lazer } \\
\text { Ter atividade de lazer } \\
\text { Não ter atividade de lazer }\end{array}$ & $\begin{array}{l}\text { Funcionamento Físico } \\
\text { Energia/Fadiga }\end{array}$ & $\begin{array}{l}1,035 \\
0,753\end{array}$ & $\begin{array}{c}- \\
0,004 \\
0,038\end{array}$ & $\begin{array}{l}2,82 \\
2,12\end{array}$ & $\begin{array}{l}1,38-5,75 \\
1,04-4,34\end{array}$ \\
\hline Tempo de hemodiálise & Função Cognitiva & $-0,014$ & 0,029 & 0,99 & $0,97-1,00$ \\
\hline $\begin{array}{l}\text { Patologia associada } \\
\text { DM }\end{array}$ & - & & - & & \\
\hline $\begin{array}{l}\text { HSA } \\
\text { DM + HAS }\end{array}$ & $\begin{array}{c}\text { Sintomas /problemas } \\
\text { Sobrecarga da doença renal } \\
\text { Bem-estar emocional }\end{array}$ & $\begin{array}{l}1,67 \mid \\
-1,590 \\
1,264\end{array}$ & $\begin{array}{l}0,032 \\
0,010 \\
0,025\end{array}$ & $\begin{array}{l}5,32 \\
0,20 \\
3,54\end{array}$ & $\begin{array}{r}1,16-24,52 \\
0,60-0,69 \\
1,17-1,06\end{array}$ \\
\hline Albumina & Funcionamento físico & $-|, 20|$ & 0,002 & 0,30 & $0,14-0,64$ \\
\hline
\end{tabular}

como as mais comprometidas. Quanto à avaliação da saúde de maneira global, os sujeitos consideraram que a sua saúde encontrava-se aproximadamente igual a de um ano atrás e a estava no meio dos extremos pior e melhor.

No presente estudo, a idade foi uma das variáveis que influenciou as dimensões 'sono', 'funcionamento físico' e 'dor', de tal forma que quanto maior a idade, menor os escores nestas dimensões. Os distúrbios do sono são prevalentes em pacientes idosos pela própria alteração fisiológica ${ }^{27}$, mas em pacientes renais crônicos este distúrbio pode ser um aspecto considerado de maior relevância ${ }^{28,29}$. A IRC, em estudo realizado para avaliar aptidão física nesta população, mostrou que, devido à limitação cardiovascular os sujeitos apresentaram dificuldades físicas para desempenhar suas atividades diárias e para cumprir as exigências do tratamento e do autocuidado $0^{30}$. Estas dificuldades serão acentuadas quanto maior a idade dos sujeitos. O limiar e a discriminação espacial para os estímulos dolorosos estão comprometidos no indivíduo idoso. As estruturas e função das unidades neurais estão alteradas nesses sujeitos e podem interferir na apreciação e expressão das queixas álgicas ${ }^{3 !}$. Isto é, com o envelhecimento ocorre um desgaste natural no organismo, o que aumenta as sensações dolorosas no idoso. Acrescido a isso, a avaliação dessa dor, sendo muito particular e subjetiva, depende do seu significado para pacientes e familiares. Os homens apresentaram escores maiores na dimensão 'dor', sugerindo então que as mulheres expressam mais queixas álgicas, o que pode ser corroborado com os estudos que avaliaram a QV em pacientes renais crônicos quanto à diferença de sexo ${ }^{32}$ e pacientes idosos ${ }^{33}$. 
Os sujeitos com segundo grau completo/incompleto apresentaram maior chance de responder que a doença renal não é uma sobrecarga em suas vidas, comparados aos sujeitos com escolaridade mais baixa. Quanto maior o nível de escolaridade, maior o acesso às informações e melhores as condições econômicas; dessa forma esses sujeitos avaliam de maneira mais assertiva os eventos traumáticos.

Observou-se também associação entre a condição de habitação boa e uma pontuação maior nas dimensões 'suporte social', 'dor', 'energia/ fadiga', evidenciando uma maior satisfação desses sujeitos com relação à QV quando comparados aos sujeitos com condição de habitação ruim. $O$ nível de vida mais elevado permite às pessoas um acesso facilitado aos tratamentos e recursos disponíveis, favorecendo assim uma melhor $\mathrm{QV}^{34}$.

Ao relacionarmos as dimensões do KDQOL-SF com 'ocupação atual', constatou que as pessoas que não trabalham apresentaram escores mais elevados no 'suporte social' e 'estímulo por parte da equipe', porém apresentaram escores menores na dimensão 'papel profissional' quando comparados aos sujeitos que trabalham com remuneração. Acredita-se que uma avaliação mais positiva quanto ao convívio social desses sujeitos seja devido ao fato de terem mais tempo para interações com familiares, amigos e os profissionais da equipe que os atendem. Estudos demonstraram uma pontuação menor dos sujeitos com IRC quando avaliaram o papel profissional20,26,35. Existe uma dificuldade dos pacientes em tratamento hemodialítico manterem seu vínculo empregatício, pois o tempo despendido com o tratamento hemodialítico gira em torno de seis horas/dia. Após as quatro horas de hemodiálise, este paciente leva aproximadamente mais duas horas para recuperação dos sintomas imediatos depois do tratamento ${ }^{36}$. Isto determina que parte dos portadores de IRC para de exercer totalmente qualquer tipo de trabalho remunerado em decorrência das limitações que a doença e o tratamento lhes impõem ${ }^{37}$.

Quando os sujeitos declararam não ter atividade de lazer, estes apresentaram escores inferiores no 'funcionamento físico', mas, por outro lado, escores superiores na dimensão 'vitalidade (energia/fadiga)'. A dimensão 'funcionamento físico' avalia a capacidade dos indivíduos em realizar atividades físicas. Dessa maneira, um escore baixo nessa dimensão explicaria a correlação com não ter atividade de lazer, já que essas atividades exigem a realização de exercícios físicos. No entanto, esses sujeitos não se sentem sem energia, esgotados ou cansados - aspectos avaliados na dimensão 'vitalidade (energia/fadiga)'.

Em relação ao tempo de hemodiálise, este mostrou associação com a 'função cognitiva', isto é, quanto maior o tempo de hemodiálise, maior as chances de os sujeitos responderem com pontuação mais elevadas na 'função cognitiva'. A dimensão 'função cognitiva' refere-se a questões que avaliam como os sujeitos se sentem em relação a sua capacidade de concentrar, discernir e reagir às situações vividas nas últimas quatro semanas. Considera-se que quanto maior o tempo de hemodiálise, maior seja a capacidade dos sujeitos em aceitar o tratamento e a doença. Um estudo que descreveu a QV dos pacientes em hemodiálise evidenciou que o paciente com maior tempo de diálise elabora, no decorrer do tratamento e do confronto perante a cronicidade da doença, respostas mais conformistas, não significando efetivamente preservação da $\mathrm{QV}^{37}$. Acrescenta-se ainda que a doença renal crônica e o tratamento hemodialítico afetam consideravelmente a QV dos pacientes, tanto nos seus parâmetros objetivos quanto subjetivos ${ }^{37}$.
Quanto à variável patologia associada, HAS mostrou associação com os 'sintomas/problemas' e 'sobrecarga da doença renal', de maneira que os escores foram mais baixos na primeira dimensão e mais elevados na segunda quando comparados aos portadores de DM. Considera-se que na HAS, a avaliação dos sujeitos de que a patologia seja uma sobrecarga menor possa ser devido ao tratamento que é menos agressivo, consistindo na maioria das vezes no monitoramento da pressão arterial e medicamentos por via oral. No entanto, quando a DM e a HAS estão associadas, o indivíduo portador de IRC avalia mais negativamente a dimensão 'bem-estar emocional'. Nesse aspecto, estes sujeitos passam por restrições referentes as doenças crônicas DM, HAS e IRC, o que os deixam com uma avaliação negativa das dimensões de sua vida.

Os valores de albumina mostraram correlaçãa com o 'funcionamento físico'. Martín et al. ${ }^{35}$, usando o KDQOL-SF na avaliação QV de pacientes de uma unidade de hemodiálise, encontraram associação negativa da albumina com 'sobrecarga da doença renal'.

\section{Conclusão}

O KDQOL-SF, na sua versão em português, mostrou propriedades psicométricas satisfatórias, o que o torna um instrumento adequado para mensurar a QV vida em portadores de IRC em tratamento hemodialítico, permitindo sua utilização para estudos que avaliem tratamentos, comparem a QV desses sujeitos entre nações e subsidiando ações intervencionistas. Os valores baixos encontrados para o $\alpha$ de Cronbach na dimensão 'papel profissional' neste estudo não compromete a aplicação do instrumento. Conhecer como esses sujeitos percebem a sua QV torna-se importante para subsidiar intervenções mais adequadas que atendam as necessidades dessa clientela.

\section{Conflito de interesse: não há}

\section{SUMMARY}

\section{ASSESSMENT OF THE BASIC PSYCHOMETRIC PROPERTIES FOR THE PORTUGUESE VERSION OF THE KDQOL-SF ${ }^{T M^{*}}$}

OBIECTIVE. To verify the reliability of the instrument Kidney Disease Quality of Life Short Form - KDQOL-SF, and correlate scores in each dimension with the sociodemographic and clinical variables.

METHOD. This is a methodological descriptive exploratory study, where the KDQOL-SF

was applied by interviewing 147 chronic renal disease patients during treatment in a hemodialysis clinic in the interior of São Paulo State. Data was processed by descriptive statistics, Cronbach's alpha and ordinal logistic regression analyses.

RESULTS. Results showed that of the 147 patients who answered the KDQOL-SF, the majority was male, with an average age of $51.8 \pm 14.2$ years and that $75.5 \%$ of them had only elementary schooling. Regarding reliability of the KDQOL-SF verified by the Cronbach's alpha, values ranged between 0.6 and 1.0 for 18 of the 19 dimensions that comprise the instrument. Only the dimension "Professional Role" showed an extremely low value. When the KDQOL-SF was correlated with sociodemographic and clinical variables, there was an association of some dimensions with age, gender, educational level and with pathologies associated to renal disease. 
Moreira CA et Al.

CONCLUSION. The KDQOL-SF was shown to be reliable to ascertain the quality of life in individuals with chronic renal failure under hemodialysis treatment. [Rev Assoc Med Bras 2009; 55(I): 22-8]

KEY wORDS: Quality of life. Renal failure. Chronic renal dialysis.

\section{REFERÊNCIAS}

I. Minayo MC, Hartz ZMA, Buss PM. Qualidade de vida e saúde: um debate necessário. Ciência e Saúde Coletiva. 2000;5:7-31.

2. Seidl EMF, Zannon CMLC. Qualidade de vida e saúde aspectos conceituais e metodológicos. Caderno Saúde Pública. 2004;20:580-8.

3. Martins LM, França APD, Kimura M. Qualidade de vida de pessoas com doença crônica. Rev Latino-Am. Enfermagem. 1996;4:5- 18.

4. Martins MRI, Cesárino CB. Qualidade de vidas de pessoas com doença renal crônica em tratamento hemodiálitico. Rev Latino-Am Enfermagem. 2005; 13:670-6.

5. Laureti RA. A mensuração da qualidade de vida. Rev Assoc Med Br. 2003; 49:36I-2.

6. Campolina AG, Ciconelli RM. Qualidade de vida e medidas de utilidade: parâmetros clínicos para tomadas de decisão em saúde. 2006; | 9: | 28-36.

7. França APD, Kimera M. Qualidade de vida de pessoas com doença crônica. Rev Latino-Am Enfermagem. 1996; 4:5-18.

8. Valderrábano F, Jofre R, Lopez-Gomes JM. Quality of life in End-Stage Renal Disease Patients. Am J Kidney Diseases. 200I; 38:443-64.

9. Souza FF, Cintra FA, Gallani MCBJ. Qualidade de vida e severidade da doença em idosos renais crônicos. Rev Bras Enferm. 2005;58:540-4.

10. Thompson DR, Meadows KA, Lewin RJP. Measuring quality of life in patients with coronary heart disease. European Heart Journal. 1998; 19:693-5.

I I. Castro M, Caiuby AVS, Draibe AS, Canziani MEF. Qualidade de vida de pacientes com insuficiência renal crônica em hemodiálise avaliada através do instrumento genérico SF-36. Rev Assoc Méd Bras. 2003; 49:245-9.

12. Duarte PS, Miyazaki MCOS, Ciconelli RM, Sesso R. Tradução e adaptação cultural do instrumento de avaliação de qualidade de vida para pacientes renais crônicos (KDQOL-SF TM). Rev Assoc Med Bras. 2003;49(4). Avaliable from:http://www.scielo.br/scielo.php.

13. Fleck MPA, Leal OF, Louzada S, Xavier M, Chachamovich E, Vieira G, et al. Desenvolvimento da versão em português do instrumento de avaliação de qualidade de vida da OMS (WHOQOL-100). Rev Bras Psiquiatria. 1999;2I(I). Avaliable from:http://www.scielo.br/scielo.php.

14. Organização Mundial de Saúde: Programa de saúde mental. Organização mundial de saúde. Genebra. Grupo WHOQOL Versão em português dos instrumentos de avaliação de qualidade de vida (WHOQOL). 1998. Avaliable from:http://www.ufrgs.br/psiq/whoqol 84.html.

15. Ciconelli RM, Ferraz MB, Santos W, Meinão I, Quaresma MR. Tradução para língua portuguesa e validação do questionário genérico de avaliação de qualidade de vida SF-36 (Brasil SF-36). Rev Bras Reumatol. 1999:39:143-50.

16. Polit DF, Hungler BP. Qualidade dos dados. In: Polit DF, Hungler BP. Fundamentos de Pesquisa em Enfermagem. $3^{\mathrm{a}}$ ed. Porto Alegre: Artes Médicas; 1995. p.199-219.

17. Fontin M-F, Nadeau M. A medida em investigação. In: Fontin M-F. O processo de investigação- da concepção à realização. $2^{a}$ ed. Loures: Lusociência; 1999. p.215 - 237.

18. LoBiondo-Wood G, Haber J. Confiabilidade e Validade. In: LoBiondoWood G, Haber J. Pesquisa em Enfermagem- Método, Avaliação Crítica e Utilização. $4^{a}$ ed. Rio de Janeiro:Guanabara-Koogan; 1998. p. I 86- 199.

19. Cronbach, LJ. Coeficient alpha and internal structure of tests. Psychometrika, 16:297-334.1951.

20. Penneger TV, Lesk M, Chopard CS, Martin PY. Assessment of health status in chronic hemodialysis patients. J Nefrol. 2003; 16:252-9.
21. Kontodimopoulos N, Niakas D. Determining the basic psychometric properties of the Greek KDQOL-SF. Quality Life Res. Available from: www.springerlink.com.

22. Molsted S, Heal J, Prescott L, Eidema KI. Reliability Testing of the Danish version of the Kidney Disease Quality of life Short Form. Scand J Trol Nephrol. 2005;39:498-502.

23. Green J, Fukuhara S, Shinzato T, Miura V, Hays RD, Tabata R, et al. Translation, cultural adaptation, and initial reliability and multitrait testing of the Kidney Disease Quality of life instrument for use in Japan. Qual Life Res. 2001;10:93-100.

24. Hays RD, Kallich JD, Mapes DL, Coons SJ, Carter WB. Development of the Kidney Disease Quality of Life (KDQOL) instrument. Qual Life Res. 1994:3:329-38.

25. Bareotei S, Molnar MZ, Almasi C,Kovacs AZ, Remport A, Szeifert L, et al. Validation of the Kidney Disease Quality of Life-Short Form questionnaire in Kidney Transplants patients. I Psichosom Res. 2006;60:495-504.

26. Kussomota L. Avaliação da qualidade de vida relacionada à saúde de pacientes em hemodiálise [tese]. Ribeirão Preto: Escola de Enfermagem de Ribeirão Preto, Universidade de São Paulo, 2005

27. Ceolin MF. Sono e Repouso. In: Duarte YAO, Diogo MJD'E. Atendimento domiciliar- Um Enfoque Gerontológico. $2^{\mathrm{a}}$ ed. São Paulo:Atheneu; 2000. p. I45-70.

28. Mucsi I, Molnar MZs, Rethelyi J,Vamos E, Csepanyi G, Tompa G. et al. Sleep disorders and illness intrusiveness in patients of chronic dialysis. Nephrol Dial Transplant. 2004;19:1815-22. Avaliable from: http:// lib.bioinfo.pl/mid.

29. Perl J, Unruh ML, Chan CT. Sleep disorders in end-stage renal disease: Markers of inadequate dialysis. Kidney Int. 2006. Available from:http:// lib.bioinfo.pl/pmid.

30. Medeiros RH, Pinent CEC. Aptidão Física do Individuo com Doença Renal Crônica. J Bras Nefrol. 2002; 24:81-7.

31. Pimenta CAM, Teixeira MJ. Dor no Idoso. In: Duarte YAO. Atendimento Domiciliar - Um Enfoque Gerontológico. $2^{\mathrm{a}}$ ed. São Paulo: Atheneu. 2000. p.373-400

32. Valderrabáno F, Sanz D, Lopez JM. Diferencias en calidad de vida en pacientes en hemodiálise en función del sexo del paciente . |999-200 I. Available from: http:www.mtas.es/mujer/mujeres/estudiuves/

33. Cunqueiro JMG, Coptes MJG, Fernando J, Borrego JF, Peracles MCS. Calidad de vida relacionada con la salud en pacientes ancioanos en hemodialises. Available from:www.revistanefrologia.com/ mostrarfille.asp.

34. Reis B. Estudo analisa a qualidade de vida com base no mundo sócio econômico. Jornal de Psiquiatria. 2003. Available from: http:// www.unifesp.br/comunicacao/jpta/ed I 77/pesquisa6.htm.

35. Martin F, Reig A, Sarró F, Ferrei R, Arenas D, Gonzalez F et al. Evolución de la calidad de vida en pacientes de una unidad de hemodialisis comnel cuestionario Kidney Disease Quality of Life-Short Form (KDQOL-SF). Dyt 2004; 25:79-92. Available from: sedyt.org/2004/ revista/numeros enteros/25 2/2502-079-0-mareh.

36. Carreira L, Marcon SS. Cotidiano e trabalho: concepções de indivíduos portadores de insuficiência renal crônica e seus familiares. Rev Latino-Am Enfermagem. 2003; I1:823-31. Available from: www.eerp.br/rlaenf.

37. Gomes CMA. Descrição da Qualidade de vida dos pacientes em hemodiálise. Rev Med. Minas Gerais. 1997;7:60-4.

Artigo recebido: 12//2/06

Aceito para publicação: 29/06/08 